13.2;15.2

\title{
Нелинейные эффекты при распылении арсенида галлия и кремния кластерными ионами висмута
}

\author{
(C) A.Б. Толстогузов ${ }^{1-3}$, П.А. Мажаров ${ }^{4}$, A.E. Иешкин ${ }^{5}$, F. Meyer ${ }^{4}$, D.J. $F u^{3}$ \\ ${ }^{1}$ Рязанский государственный радиотехнический университет им. В.Ф. Уткина, Рязань, Россия \\ ${ }^{2}$ Centre for Physics and Technological Research, Universidade Nova de Lisboa, Caparica, Portugal \\ 3 Key Laboratory of Artificial Micro- and Nanostructures of Ministry of Education and Hubei Key Laboratory of Nuclear Solid \\ Physics, School of Physics and Technology, Wuhan University, Wuhan, China \\ ${ }^{4}$ Raith GmbH, Dortmund, Germany \\ 5 Московский государственный университет им. М.В. Ломоносова, Москва, Россия \\ E-mail: a.tolstoguzov@fct.unl.pt
}

Поступило в Редакцию 8 ноября 2021 г.

В окончательной редакции 1 декабря 2021 r.

Принято к публикации 2 декабря 2021 г.

Проведено экспериментальное исследование влияния энергии и количества атомов в бомбардирующих ионах $\mathrm{Bi}_{n}^{+}(n=1-4)$ на коэффициент распыления GaAs. Показано, что удельный коэффициент распыления $Y_{s p}$ неаддитивно увеличивается с ростом $n$ и кинетической энергии $E_{s p}$, приходящейся на один атом в бомбардирующем ионе, и эффективность передачи энергии от бомбардирующих ионов к атомам мишени также возрастает с ростом $n$. Проведено сравнение с полученными ранее результатами для $\mathrm{Si}$-мишеней.

Ключевые слова: ионное распыление, коэффициент неаддитивности, кластерные ионы, висмут.

DOI: 10.21883/PJTF.2022.05.52150.19071

В настоящее время сфокусированные пучки атомных и кластерных ионов тяжелых металлов, в первую очередь $\mathrm{Sn}, \mathrm{Au}, \mathrm{Pb}$ и $\mathrm{Bi}$, получаемые с помощью электрогидродинамических (жидкометаллических) ионных источников [1], используются для решения широкого круга фундаментальных и прикладных задач в микро- и нанотехнологиях, аэрокосмической технике и микрозондовом анализе. Применение кластерных ионов в ионнолучевой литографии и наноструктурировании позволяет увеличить коэффициент распыления. Большое число работ (см., например, [2-8]) посвящено компьютерному моделированию процессов распыления и расчетам коэффициентов распыления. Вместе с тем публикаций об экспериментальных измерениях коэффициентов распыления кластерными ионами, а тем более данных о сравнительной эффективности распыления атомными и кластерными ионами в одинаковых экспериментальных условиях значительно меньше $[9,10]$. В нашей предыдущей работе [11] проведено исследование влияния энергии и количества атомов $n$ в бомбардирующих ионах $\mathrm{Bi}_{n}^{+}(n=1-4)$ на коэффициент распыления $\mathrm{Si}$. Было установлено, что удельный коэффициент распыления $Y_{s p}$, который показывает, сколько атомов мишени распыляется на один атом, входящий в состав кластерного иона, неаддитивно увеличивается с ростом $n$ и кинетической энергии $E_{s p}$, приходящейся на один атом в бомбардирующем ионе. В качестве фактора неаддитивности $k$ было предложено использовать тангенс угла наклона прямой линии, аппроксимирующей зависимость $Y_{s p}(n)$ при одинаковом значении $E_{s}$. Для $\mathrm{Si}$ при облучении $10 \mathrm{keV}$ ионами $\mathrm{Bi}_{n}^{+}(n=1-4)$ этот фактор оказался равным $0.4 \pm 0.1$. В настоящей работе мы расширили круг исследованных материалов за счет двухкомпонентного соединения $\mathrm{GaAs}$ - важного полупроводникового материала, третьего после кремния и германия по масштабам промышленного использования.

Эксперименты проводились на коммерческом ионнолучевом литографе VELION (Raith Nanofabrication, Germany [12]), технические характеристики можно найти в [1]. Масс-сепарированные пучки атомных $\mathrm{Bi}^{+}(n=1)$ и кластерных ионов $\mathrm{Bi}_{n}^{+}(n=2-4)$ [13] направлялись по нормали к поверхности мишени. Ускоряющее напряжение изменялось в диапазоне $10-40 \mathrm{kV}$ с шагом в $10 \mathrm{kV}$, при этом диаметр пучка на мишени для всех типов ионов не превышал $100 \mathrm{~nm}$ (FWHM), a ионный ток был в пределах $0.02-1 \mathrm{nA}$ в зависимости от энергии и числа атомов в кластерном ионе. Долговременная стабильность ионного тока была лучше $1 \%$ за $7 \mathrm{~h}$ непрерывной работы, и величина ионного тока на мишени автоматически контролировалась каждые $10 \mathrm{~s}$.

Полный коэффициент распыления $Y$ определялся исходя из объема распыленного вещества $V$, равного произведению площади на глубину кратера, и полной дозы бомбардирующих ионов $F_{\text {ion }}$ :

$$
Y=\frac{V N}{F_{i o n}}
$$

Здесь $N$ - атомная плотность распыляемого вещества (мишени),

$$
F_{\text {ion }}=\frac{I t}{e}
$$

где $I$ - ионный ток на мишени, $t-$ время распыления, $e$ - элементарный заряд. 
Помимо полного коэффициента распыления $Y$ мы определяли удельный коэффициент $Y_{s p}$ (specific sputter yield), равный $Y / n$, т.е. в формуле (1) вместо полной ионной дозы использовалась полная доза бомбардирующих атомов $F_{a t}=n F_{i o n}$. Необходимо отметить, что в этом методе, получившем название Volume Loss Method (VLM) [14], серьезные требования предъявляются к колонне первичного ионного пучка (ионной пушке), которая должна генерировать остросфокусированные и масссепарированные пучки атомных и кластерных ионов с одинаковой энергией $E_{s}$. Выполнение этого требования необходимо для надежного разделения вкладов в коэффициент распыления от энергии и от числа атомов в бомбардирующем ионе. Кроме того, плотность ионного тока и доза бомбардирующих частиц должны быть достаточными для формирования кратера, линейные размеры и глубину которого можно точно измерить с помощью профилометра. В наших экспериментах использовался контактный профилометр Veeco DEKTAK 150 с вертикальным разрешением $0.1 \mathrm{~nm}$. Измерения проводились в трех точках, произвольно выбранных на дне каждого кратера, погрешность измерений не превышала $\pm 0.5 \mathrm{~nm}$.

Всего было приготовлено 16 кратеров при облучении $\mathrm{GaAs}$ ионами $\mathrm{Bi}_{n}^{+}(n=1-4)$ с энергиями $10,20,30$ и $40 \mathrm{keV}$ для каждого типа ионов. Глубина и площадь кратеров составляли $65-1425 \mathrm{~nm}$ и $400-700 \mu \mathrm{m}^{2}$ соответственно. Для всех типов ионов и их энергий линейные размеры кратеров значительно превышали 100 диаметров ионного пучка, что вместе с быстрым сканированием этого пучка, направленного по нормали к поверхности мишени, обеспечивало формирование кратеров с вертикальными стенками и однородным дном. Кроме того, коэффициенты распыления атомарными ионами висмута оценивались с помощью программы TRIM [15], при этом состав образца выбирался равным номинальному, т.е. действовало приближение мишени, не возмущенной ионным облучением.

На рис. 1 представлены удельные коэффициенты распыления $Y_{s p}(n)$ арсенида галлия ионами $\mathrm{Bi}_{n}^{+}(n=1-4)$ в зависимости от энергии $E_{s p}$. По тангенсам углов наклона линейных аппроксимаций этих зависимостей можно судить об эффективности ионного распыления. Для GaAs значения тангенсов составили $0.19\left(\mathrm{Bi}^{+}\right)$и $3.2\left(\mathrm{Bi}_{4}^{+}\right)$. Для сравнения в случае кремния эти величины равнялись $0.09\left(\mathrm{Bi}^{+}\right), 0.17\left(\mathrm{Bi}_{2}^{+}\right)$и $0.25\left(\mathrm{Bi}_{3}^{+}\right)$[11]. Полный коэффициент распыления $Y$ в наших экспериментах для $40 \mathrm{keV}$ ионов $\mathrm{Bi}_{4}^{+}$был равен 125 (GaAs) и $15(\mathrm{Si})[11]$.

На рис. 2 представлены зависимости $Y_{s p}(n)$ для $\mathrm{GaAs}$ и $\mathrm{Si}$ при облучении ионами $\mathrm{Bi}_{n}^{+}$с энергией $10 \mathrm{keV} /$ atom. Тангенсы углов наклона прямых линий, аппроксимирующих эти зависимости, равны $2.8 \pm 0.2$ и $20(\mathrm{GaAs})$ и $0.4 \pm 0.1(\mathrm{Si})$ [11]. Мы использовали значения этих тангенсов в качестве факторов неаддитивности $k$, т.е. как количественную характеристику вклада нелинейных процессов в механизм(ы) распыления. Отметим, что в этом случае для вычисления $k$ -

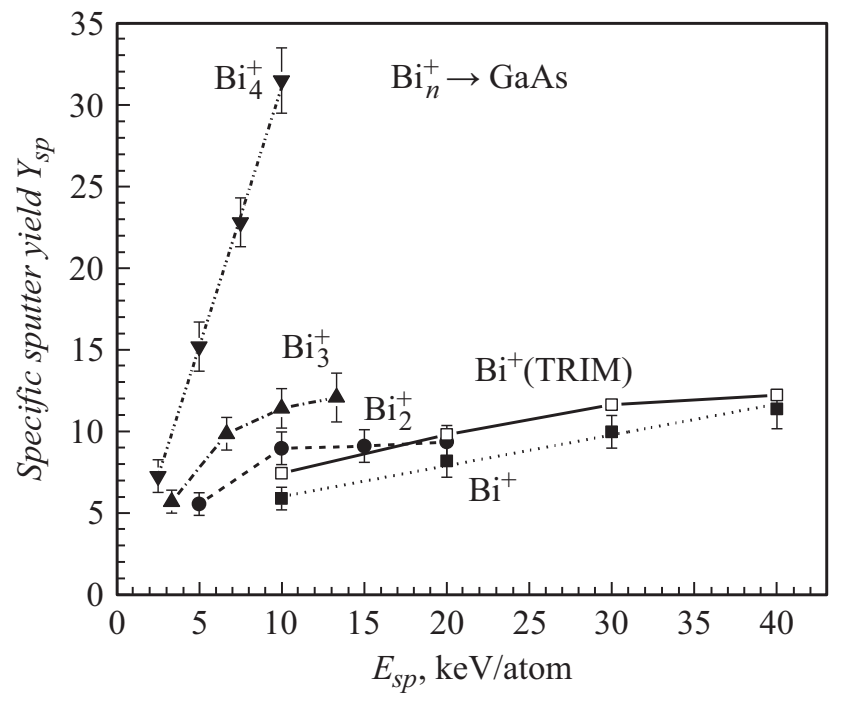

Рис. 1. Зависимости удельных коэффициентов распыления $\mathrm{GaAs}$ ионами $\mathrm{Bi}_{n}^{+}(n=1-4)$ от энергии, приходящейся на один атом в бомбардирующем ионе, и линейные аппроксимации этих зависимостей для $\mathrm{Bi}^{+}$и $\mathrm{Bi}_{4}^{+}$. Для сравнения приведена расчетная зависимость коэффициента распыления, полученная с помощью TRIM для ионов $\mathrm{Bi}^{+}$(сплошная линия со светлыми квадратами).

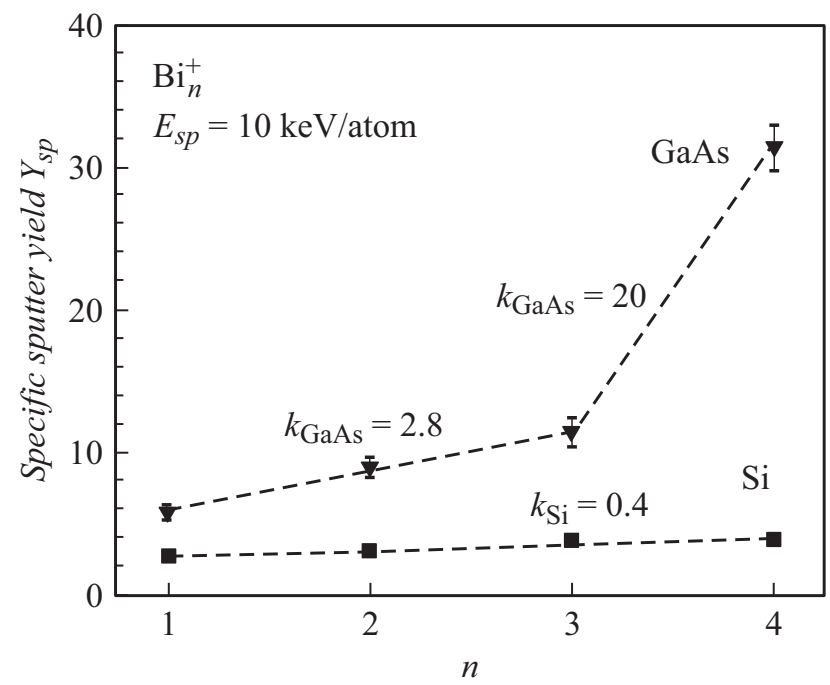

Рис. 2. Зависимости удельных коэффициентов распыления $\mathrm{GaAs}$ и $\mathrm{Si}[11]$ ионами $\mathrm{Bi}_{n}^{+}$с энергией $10 \mathrm{keV} /$ atom от числа атомов $n$, входящих в состав кластерного иона. Значения факторов неаддитивности $k$, равные тангенсам углов наклона прямых, аппроксимирующих экспериментальные зависимости $Y_{s p}(n)$, приведены на поле рисунка.

фактора нет необходимости привлекать дополнительные компьютерные расчеты, как, например, это было сделано в [9].

В рамках краткого сообщения нет возможности детально обсудить все механизмы, ответственные за увеличение $Y_{s p}$ с ростом числа атомов $n$ в бомбардирующем ионе $\mathrm{Bi}_{n}^{+}(n=1-4)$ и удельной энергии $E_{s p}$. Вероятнее 
всего, наибольший вклад вносят тепловые пики (thermal spikes) (см. работы [16,17] и ссылки в них), в объеме которых реализуется нелинейный режим распыления, сопровождаемый локальным плавлением и испарением вещества, причем эффективность этих процессов возрастает с ростом $n$ и $E_{s p}$ (рис. 1). Также было установлено (рис. 2), что при одинаковом значении $E_{s p}$ увеличение числа атомов в бомбардирующем ионе ведет к неаддитивному росту $Y_{s p}$. Факторы неаддитивности $k$ оказались существенно разными для материалов, исследованных в настоящей работе и [11]. Это может быть связано с ограничением размеров тепловых пиков в GaAs по сравнению с Si из-за меньших пробегов первичных ионов и атомов отдачи в этом материале. Также нельзя исключить влияние температурной проводимости, которая у $\mathrm{GaAs}$ более чем в 3 раза меньше, чем у $\mathrm{Si}$ [18], что может способствовать локализации тепловых пиков в меньших объемах, приводить к более высокой температуре и, как следствие, существенно влиять на распыление атомов мишени в нелинейном режиме.

Известно, что GaAs плавится, а затем испаряется инконгруэнтно (с разложением), а именно с образованием жидкого галлия и молекул газообразного мышьяка [19]. Вероятнее всего, изменение состава GaAs в объеме теплового пика оказывает влияние на зависимости $Y_{s p}\left(E_{s p}\right)$, и для бомбардирующих ионов $\mathrm{Bi}_{2}^{+}$и $\mathrm{Bi}_{3}^{+}$по сравнению с $\mathrm{Bi}^{+}$и $\mathrm{Bi}_{4}^{+}$наблюдается переходный режим (рис. 1), который сопровождается изменением линейного характера зависимости $Y_{s p}\left(E_{s p}\right)$. Резкое возрастание фактора неаддитивности при переходе к ионам $\mathrm{Bi}_{4}^{+}$(рис. 2) также может быть следствием возрастания влияния эффекта инконгруэнтности на эффективность распыления.

На рис. 3 приведены зависимости полных коэффициентов распыления $\mathrm{Si}$ (по данным [11]) (a) и GaAs (b) от ядерных потерь энергии ионов висмута для кластеров с разным значением $n$. При расчете потерь энергии кластера вклады отдельных атомов считались независимыми [20,21], т. е.

$$
\left(\frac{d E}{d x}(n, E)\right)_{n u c l}=n\left(\frac{d E}{d x}\left(1, \frac{E}{n}\right)\right)_{n u c l} .
$$

На этом же рисунке штриховой линией показана зависимость, полученная моделированием в TRIM при энергиях, меньших максимума упругих потерь, и экстраполяцией этой зависимости в область больших энергий. Эта линия приближенно соответствует вкладу линейной составляющей распыления. Разница между экспериментальными точками и этой линией для GaAs значительно больше, чем при распылении $\mathrm{Si}$.

Таким образом, в работе было установлено, что фактор неаддитивности распыления больше для $\mathrm{GaAs}$, чем для $\mathrm{Si}$. Также показано, что если для кремния зависимость коэффициентов распыления от упругих потерь энергии практически совпадает для кластеров с разными $n$, то для арсенида галлия наблюдаются заметные различия между кластерами. Эта особенность может быть
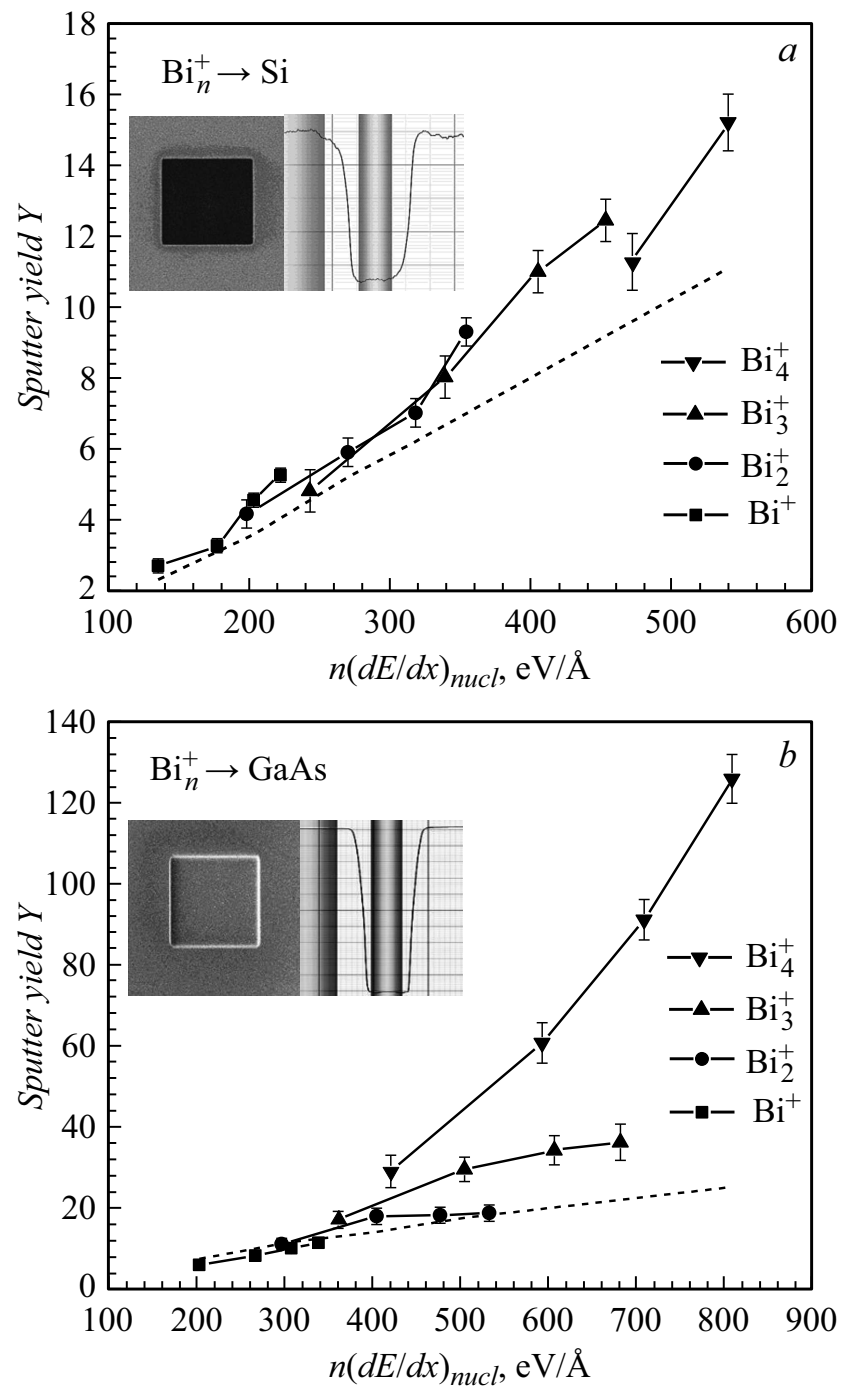

Pис. 3. Полные коэффициенты распыления $\mathrm{Si}$ (по данным [11]) (a) и $\mathrm{GaAs}(b)$ ионами $\mathrm{Bi}_{n}^{+}$в зависимости от ядерных потерь энергии кластера висмута. Штриховой линией показана аппроксимация расчетных данных, полученных с помощью TRIM. На вставках приведены изображения кратеров распыления, полученные с помощью литиевого ионного микроскопа, и профили этих кратеров, измеренные с помощью профилометра Veeco DEKTAK 150.

связана с эффектом инконгруэнтности при плавлении и испарении арсенида галлия.

\section{Финансирование работы}

Работа выполнена в рамках государственного задания Министерства науки и высшего образования РФ (FSSN-2020-0003).

\section{Конфликт интересов}

Авторы заявляют, что у них нет конфликта интересов. 


\section{Список литературы}

[1] П.А. Мажаров, В.Г. Дудников, А.Б. Толстогузов, УФН, 190 (12), 1293 (2020). DOI: 10.3367/UFNr.2020.09.038845 [P.A. Mazarov, V.G. Dudnikov, A.B. Tolstoguzov, Phys. Usp., 63 (12), 1219 (2020). DOI: 10.3367/UFNe.2020.09.038845].

[2] A.E. Ieshkin, A.V. Nazarov, A.A. Tatarintsev, D.S. Kireev, A.D. Zavilgelsky, A.A. Shemukhin, V.S. Chernysh, Surf. Coat. Technol., 404, 126505 (2020).

DOI: $10.1016 /$ j.surfcoat.2020.126505

[3] Д.С. Мелузова, П.Ю. Бабенко, А.Н. Зиновьев, А.П. Шергин, Письма в ЖТФ, 46 (24), 19 (2020). DOI: 10.21883/PJTF.2020.24.50422.18487 [D.S. Meluzova, P.Yu. Babenko, A.N. Zinoviev, A.P. Shergin, Tech. Phys. Lett., 46 (12), 1227 (2020). DOI: 10.1134/S1063785020120226].

[4] V.N. Popok, J. Samela, K. Nordlund, V.P. Popov, Phys. Rev. B, 85 (3), 033405 (2012). DOI: 10.1103/PhysRevB.85.033405

[5] M. Lindenblatt, R. Heinrich, A. Wucher, B.J. Garrison, J. Chem. Phys., 115 (18), 8643 (2001). DOI: $10.1063 / 1.1404982$

[6] C. Anders, K.-H. Heinig, H.M. Urbassek, Phys. Rev. B, 87 (24), 245434 (2013). DOI: 10.1103/PhysRevB.87.245434

[7] R.J. Paruch, Z. Postawa, B.J. Garrison, J. Vac. Sci. Technol. B, 34 (3), 03H105 (2016). DOI: 10.1116/1.4940153

[8] P.A. Karaseov, K.V. Karabeshkin, A.I. Titov, M.W. Ullah, A. Kuronen, F. Djurabekova, K. Nordlund, G.M. Ermolaeva, V.B. Shilov, J. Phys. D: Appl. Phys., 50 (50), 505110 (2017). DOI: $10.1088 / 1361-6463 / a a 97 a b$

[9] A.V. Samartsev, A. Duvenbeck, A. Wucher, Phys. Rev. B, 72 (11), 115417 (2005). DOI: 10.1103/PhysRevB.72.115417

[10] L. Bischoff, W. Pilz, P. Mazarov, A.D. Wieck, Appl. Phys. A, 99 (1), 145 (2010). DOI: 10.1007/s00339-010-5597-0

[11] A. Tolstogouzov, P. Mazarov, A.E. Ieshkin, S.F. Belykh, N.G. Korobeishchikov, V.O. Pelenovich, D.J. Fu, Vacuum, 188, 110188 (2021). DOI: $10.1016 /$ j.vacuum.2021.110188

[12] https://www.raith.com/ (12/2021)

[13] W. Pilz, N. Klingner, L. Bischoff, P. Mazarov, S. Bauerdick, J. Vac. Sci. Technol. B, 37 (2), 021802 (2019). DOI: $10.1116 / 1.5086271$

[14] P. Schneider, L. Bischoff, J. Teichert, E. Hesse, Nucl. Instrum. Meth. Phys. Res. B, 117 (1-2), 77 (1996). DOI: $10.1016 / 0168-583 X(96) 00273-X$

[15] http://www.srim.org/ (12/2021).

[16] P. Sigmund, C. Claussen, J. Appl. Phys., 52 (2), 990 (1981). DOI: $10.1063 / 1.328790$

[17] Yu. Kudriavtsev, R. Asomoza, A. Hernandez, D.Yu. Kazantsev, B.Ya. Ber, A.N. Gorokhodv, J. Vac. Sci. Technol., 38 (5), 053203 (2020). DOI: 10.1116/6.0000262

[18] Physics of semiconductor devices, 3rd ed., ed by S.M. Sze, K.K. Ng (Wiley, Hoboken, 2007).

[19] C.Y. Lou, G.A. Somorjai, J. Chem. Phys., 55 (9), 4554 (1971). DOI: $10.1063 / 1.167678$

[20] V.I. Shulga, P. Sigmund, Nucl. Instrum. Meth. Phys. Res. B, 47 (3), 236 (1990). DOI: 10.1016/0168-583X(90)90751-F

[21] S. Bouneau, A. Brunelle, S. Della-Negra, J. Depauw, D. Jacquet, Y. Le Beyec, M. Pautrat, M. Fallavier, J.C. Poizat, H.H. Andersen, Phys. Rev. B, 65 (14), 144106 (2002). DOI: 10.1103/PhysRevB.65.144106 